\title{
A Retrieved Sparganum of Spirometra erinaceieuropaei from a Korean Man during Mechanical Thrombectomy
}

\author{
Yang-Ha Hwang', Wonsoo Son ${ }^{2,3}$, Yong-Won Kim,3, Dong-Hun Kang ${ }^{2,3}$, Hyun-Ha Chang ${ }^{4}$, \\ Youn-Kyoung Goo ${ }^{5, *}$, Yeonchul Hong ${ }^{5}$, Dong-II Chung ${ }^{5}$
}

\begin{abstract}
${ }^{1}$ Department of Neurology, Kyungpook National University Hospital, School of Medicine, Kyungpook National University, Daegu 41944, Korea; 2Department of Neurosurgery, Kyungpook National University Hospital, School of Medicine, Kyungpook National University, Daegu 41944, Korea; ${ }^{3}$ Department of Radiology, Kyungpook National University Hospital, School of Medicine, Kyungpook National University, Daegu 41944, Korea; ${ }^{4}$ Department of Internal Medicine, School of Medicine, Kyungpook National University, Daegu 41944, Korea; ${ }^{5}$ Department of Parasitology and Tropical Medicine, School of Medicine, Kyungpook National University, Daegu 41944, Korea
\end{abstract}

\begin{abstract}
Human sparganosis is a zoonotic disease caused by infection and migration of the plerocercoid of Spirometra spp. Although sparganosis were reported from most parts of the body, the sparganum parasitizing inside cerebral artery is remarkably uncommon. We report a case of cerebral intravascular sparganosis in an elderly patient with acute ischemic stroke who was diagnosed by retrieving sparganum during mechanical thrombectomy. Finally, the parasites were identified as Spirometra erinaceieuropaei using multiplex PCR and cox1 gene sequencing.
\end{abstract}

Key words: Spirometra erinaceieuropaei, intravascular, sparganosis, stroke

\section{INTRODUCTION}

Human sparganosis is a zoonotic disease caused by infection and migration of the plerocercoid of Spirometra spp. Human is almost always the intermediate host, rarely infected with developing intraintestinal adult worms $[1,2]$. The routes of sparganum infection involve either drinking water contaminated with procercoid-infected copepods or consumption of undercooked meat of plerocerciod-infected snakes or frogs. The infection can occur by placing poultices that use the skin of infected snakes or frogs on applied to inflamed eyes, skin and teeth [3-5].

The clinical presentations of sparganosis are widely varied and dependent on location of the infection. The larvae invade throughout the body, including the brain, spine, eyes, skin, lungs, abdominal viscera, and genitourinary tract, and can live in humans for up to 20 years [2]. Central nervous system infection can present with headache, seizure, confusion, weakness, and/or paresthesia, depending on location of the larvae

- Received 9 December 2019, revised 29 April 2020, accepted 5 May 2020.

*Corresponding author (kuku1819@knu.ac.kr)

(c) 2020, Korean Society for Parasitology and Tropical Medicine

This is an Open Access article distributed under the terms of the Creative Commons

Attribution Non-Commercial License (https://creativecommons.org/licenses/by-nc/4.0) which permits unrestricted non-commercial use, distribution, and reproduction in any

medium, provided the original work is properly cited. and migrating path [6]. Cerebral sparganosis is a rare complication of the infection, although there have been a few reports in Republic of Korea, Japan, Thailand, and China [7,8]. In the Republic of Korea, cerebral hemorrhage and symptoms of spinal cord involvement, such as back pain and weakness, resulted from the sparganum infection have been reported $[9,10]$. However, the alive sparganum inside the cerebral vessels, which could be a source of acute ischemic stroke have not been found in human brain to date.

The species of the genus Spirometra, including S. erinaceieuropaei, S. decipiens, S. mansoni, S. ranarum, and S. mansonoides occasionally reside in human as paratenic or intermediate hosts [11-15]. After the Korean human sparganosis case in 1924 has been named as Sparganum mansoni, 2 human cases infected with $S$. erinaceieuropaei were reported based on morphological study in 1984 [16]. The morphological identification of Spirometra species infected in human host is difficult because they usually found as plerocercoid that lacks distinguishing characteristics. Lately, molecular approaches to identify Spirometra species have been introduced in human spargana cases, revealing that $S$. erinaceieuropaei and S. decipiens cause human sparganosis in Korea [17]. However, S. erinaceieuropaei did not found in terrestrial snakes, the known infectious source of spargana to human, collected from Republic of Korea and China [18]. After those previous studies, molecular diagnosis 
of sparganosis in species level becomes more important to make clear its life cycle and transmission route of this infectious disease. Therefore, we report here an intravascular sparganosis retrieved during endovascular mechanical thrombectomy for the treatment of acute ischemic stroke due to left middle cerebral artery occlusion in an elderly male and identified S. erinaceieuropaei using multiplex polymerase chain reaction (PCR) and cytochrome c oxidase subunit 1 ( $\operatorname{cox} 1)$ gene sequencing.

\section{CASE RECORD}

An 89-year-old man was admitted to the Emergency Center, Kyungpook National University Hospital on August 17, 2018 due to right side weakness and altered consciousness after one hour of symptom onset. He had medical history of hypertension and had been taking medicines for the hypertension. According to his wife, he had not had uncooked frog or water from the ponds. Routine laboratory test results were unremarkable, and no eosinophilia was noted. Neurological examination revealed global aphasia, right facial palsy of central type, right hemiparesis with Medical Research Council grade
$1 / 5$ in the right upper extremity and $1 / 5$ right lower extremity, right homonymous hemianopsia, and Babinski sign with a National Institutes of Health Stroke Scale score of 22.

Initial brain computed tomography (CT) and supra-aortic $\mathrm{CT}$ angiography revealed subtle early ischemic changes on the left temporal area and acute occlusion of left middle cerebral artery (Fig. 1A). Subsequent diffusion-weighted imaging was performed to define the extent of ischemic lesions and to guide acute stroke therapies, which showed fuzzy hyperintensities on the left fronto-temporo-parietal area including left basal ganglia (Fig. 1B). Considering his clinical and imaging status, endovascular mechanical thrombectomy was performed to reperfuse the occluded vessel after $1.5 \mathrm{hr}$ from symptom onset. Using contact aspiration and stent retriever techniques, partial reperfusion of occluded vessel was achieved (Fig. 1C, D). During mechanical thrombectomy, multiple fragmented whitish or reddish materials were retrieved from the occluded vessel. Among the retrieved materials, one whitish long segment material was mobile (Fig. 1E). To identify species, these retrieved materials were sent to the Department of Parasitology and Tropical medicine. At one day after index stroke, follow-up brain CT revealed hypodensities on left fron-
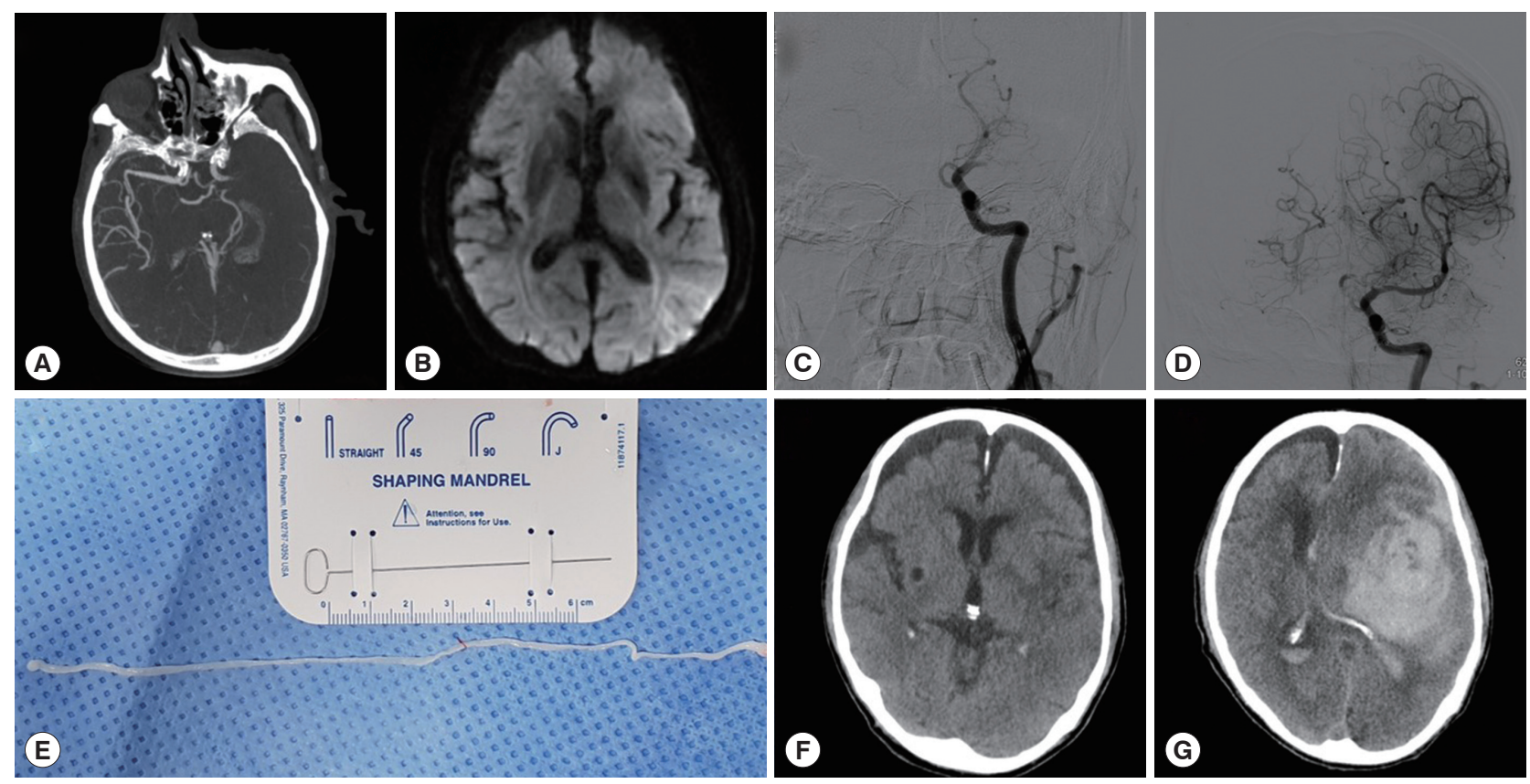

Fig. 1. Findings of an 89-year-old male with acute ischemic stroke. (A) Initial supra-aortic CT angiography showing acute occlusion of left middle cerebral artery. (B) Subsequent diffusion-weighted imaging at same day. (C) Initial left carotid angiography for endovascular mechanical thrombectomy. (D) Partial reperfusion of occluded artery was achieved. (E) One whitish long worm with sluggish movement. (F) Follow-up brain CT image at day 1. (G) After neurological worsening, a follow-up brain CT image showing massive parenchymal hematoma on the affected hemisphere. 
to-temporal area including left basal ganglia without any evidence of hemorrhagic conversions (Fig. 1F). During hospital stay, his neurological status was unchanged compared to baseline status despite the partial reperfusion of occluded vessels. At 15-days after index stroke, he suffered massive intracerebral hemorrhage on the affected hemisphere (Fig. 1G), and he died at the same day despite the medical management.

In order to identify Spirometra species, molecular analyses using multiplex PCR and partial sequencing of cox 1 were performed in Department of Parasitology and Tropical medicine. Genomic DNA was extracted from the larva of Spirometra with QIAamp DNA Mini kit (Qiagen, Hilden, Germany) according to the manufacturer's instructions. Multiplex PCR on cox 1 gene was performed with reference to the previous reports with some modifications $[17,18]$. Briefly, the forward primer, Se/Sd-7963F (5'-ACG TGG TTT GTG GTG GCT CAT TTT-3'), for the conserved sequence of both Spirometra species, and 2 specific primers for $S$. decipiens (Sd-8584R, 5'-GTA TCA AGT TGG TTA GGA AGT TAA-3') and for S. erinaceieuropaei (Se8344R, 5' -ATG ATA GGG TAT AGG TGA CCA-3') were employed for multiplex PCR using ExTaq DNA polymerase (Takara, Osaka, Japan). Thermal cycles were as following: initial denaturation at $98^{\circ} \mathrm{C}$ for $3 \mathrm{~min}$, followed by 30 cycles of denaturation at $98^{\circ} \mathrm{C}$ for $30 \mathrm{sec}$, annealing at $55^{\circ} \mathrm{C}$ for $30 \mathrm{sec}$ and extension at $72^{\circ} \mathrm{C}$ for $30 \mathrm{sec}$, and a final extension at $72^{\circ} \mathrm{C}$ for $10 \mathrm{~min}$. For reference, genomic DNA of S. erinaceieuropaei and $S$. decipiens were obtained from Parasite Resource Bank, Republic of Korea. A multiplex PCR amplicon of current sample showed a band close to $S$. erinaceieuropaei (Fig. 2). This cox 1 sequence of current specimen had identity $99.4 \%$ to S. erina-

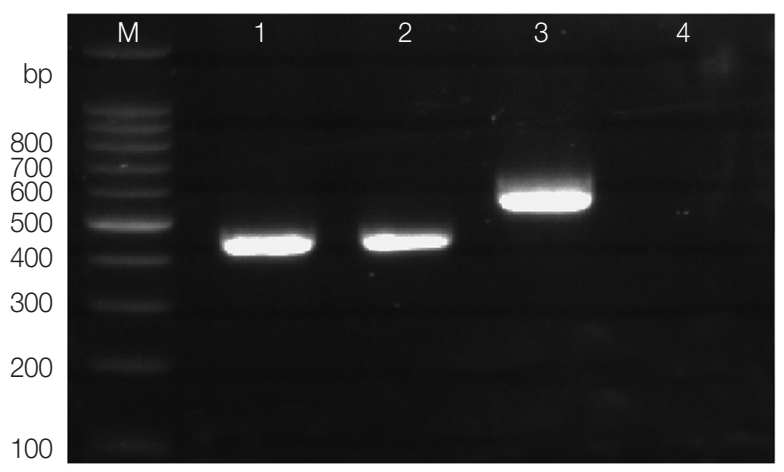

Fig. 2. Multiplex PCR of retrieved sparganum from a patient. Lane M, DNA size marker. Lane 1, genomic DNA of retrieved sparganum of present patient. Lane 2, genomic DNA of S. erinaceieuropaei. Lane 3, genomic DNA of S. decipiens. Lane 4, negative control. ceieuropaei (KJ599680) and 90.2\% to S. decipiens (KJ599679). Taken together, molecular analyses on this cerebral sparganosis revealed an infection with $S$. erinaceieuropaei.

\section{DISCUSSION}

Cerebral sparganosis is a rare complication of Spirometra spp. infection caused by their plerocercoid invading and living in the brain. Symptoms of the cerebral sparganosis are complex and diverse depending on location of the parasite in the brain. The patients present with focal neurological dysfunction including chronic paroxysmal headache, epilepsy, increased intracranial pressure, disturbance of consciousness, limb numbness and visual impairment [19]. The neurological symptoms were evoked by inflammatory reaction and granuloma formation around the spargana, as well as petechial hemorrhage and hematoma due to capillary or venous injury by the migrating worm $[9,20,21]$. Among the cases of the cerebral sparganosis, the case that the plerocercoid is parasitized in brain artery are significantly unique. Previously, 2 cases of sparganum infection related to cerebral infarction were reported to date; however, none of them found the parasite inside the artery. One case reported in 1951 by Takeuchi was the sparganosis diagnosed during autopsy in cerebral infarction patient, and another one was cerebral infarction induced by vasculitis possibly caused by sparganum mass [22,23]. It is not clear how the worm is migrated in cerebral artery. In general, when a patient gets infected, the larvae enter the abdominal cavity by passing through the alimentary canal. Then they further migrate into the diaphragm and mediastinum to reach the neck. At last, they pass through the foramen magnum and enter the brain [24]. The larvae may be incidentally entered into artery and migrated to brain during the process. Nevertheless, the location in the cerebral artery was unusual for a sparganum, as was the size of about $13 \mathrm{~cm}$.

The clinical manifestation and epidemiological history of Spirometra infection are often not specific for the sparganosis diagnosis. Therefore, characteristic signs on brain computed tomography (CT) and magnetic resonance imaging (MRI) have been used to make an accurate diagnosis. It was first reported that MRI on sparganosis showed white matter degeneration, as indicated by weak T1WI signal and strong T2WI signal in the low density CT [20]. Rate of accurate diagnosis of cerebral sparganosis could be increased from 0 to $11.8 \%$ and $28.6 \%$ with multiple follow-up examinations [25]. In addi- 
tion, the most typical MRI manifestation was tunnel-like or rope-like, with the most common being bead-shaped enhancement [26]. CT findings of cerebral sparganosis usually demonstrated presence of the white matter hypoattenuation with dilatation of adjacent ventricle, irregular or nodular enhancing lesion, small punctate calcification, and a change in the location of the enhancing nodule on the follow-up CTs $[20,27]$. In present case, those signs of sparganosis shown in CT images were not critically appeared, which may be because the sparganum manifested only vessel occlusion, but not the inflammatory reaction or hemorrhage in brain in current case.

In general, S. erinaceieuropaei was considered only Spirometra species in Republic of Korea. Therefore, a differentiation of species level using molecular method has not been considered. However, recent study suggested that $S$. decipiens is another cause of human sparganosis in Republic of Korea. Moreover, it was also reported that S. erinaceieuropaei may have other intermediate host, not snakes $[17,18]$. These previous observations support that molecular diagnosis of species level of sparganosis is needed to understand epidemiology of sparganosis. This present specimen was identified as S. erinaceieuropaei based on multiplex PCR and cox 1 sequencing. According to his wife, there are few possibility that the patient was infected with the sparganum by frogs or snake consumption. The only $S$. decipiens was found in snakes in the previous study [18]. Therefore, it seems that the S. erinaceieuropaei has infection route different from $S$. decipiens. To clarify the transmission route and life cycle of $S$. erinaceieuropaei, further studies using a number of samples from humans and wild animals (possible final hosts) would be required.

\section{CONFLICT OF INTEREST}

We have no conflict of interest related to this work.

\section{REFERENCES}

1. Liu Q, Li MW, Wang ZD, Zhao GH, Zhu XQ. Human sparganosis, a neglected food borne zoonosis. Lancet Infect Dis 2015; 15: 1226-1235.

2. Lu G, Shi DZ, Lu YJ, Wu LX, Li LH, Rao LY, Yin FF. Retrospective epidemiological analysis of sparganosis in mainland China from 1959 to 2012. Epidemiol Infect 2014; 142: 2654-2661.

3. Kobayashi H. On the animal parasites in Chosen (Korea). Second report. Acta Med Keijo 1928; 11: 109-124.

4. Weinstein PP, Krawczyk HJ, Peers JH. Sparganosis in Korea. Am J
Trop Med Hyg 1954; 3: 112-129.

5. Seo BS, Rim HJ, Yoon JJ, Lee DJ. A case report of sparganosis. Korean J Parasitol 1964; 2: 179-182 (in Korean).

6. Hong D, Xie H, Zhu M, Wan H, Xu R, Wu Y. Cerebral sparganosis in mainland Chinese patients. J Clin Neurosci 2013; 20: 1514-1519.

7. Anantaphruti MT, Nawa Y, Vanvanitchai Y. Human sparganosis in Thailand: an overview. Acta Trop 2011; 118: 171-176.

8. Shin EH, Guk SM, Kim HJ, Lee SH, Chai JY. Trends in parasitic diseases in the Republic of Korea. Trends Parasitol 2008; 24: 143-150.

9. Jeong SC, Bae JC, Hwang SH, Kim HC, Lee BC. Cerebral sparganosis with intracerebral hemorrhage: a case report. Neurology 1998; 50: 503-506.

10. Kwon JH, Kim JS. Sparganosis presenting as a conus medullaris lesion: case report and literature review of the spinal sparganosis. Arch Neurol 2004; 61: 1126-1128.

11. Muller JF. Spargana in Natrix. Science 1937; 85: 519-520.

12. Faust EC, Campbell HE, Kellogg CR. Morphological and biological studies on the species of Diphyllobothrium in China. Am J Epidemiol 1929; 9: 560-583.

13. Yamaguti S. Systema Helminthum. Vol. II. The Cestodes of Vertebrates. New York, USA. Interscience Publishers. 1959, pp 358361.

14. Wardle RA, McLeod JA. The Zoology of Tapeworms. Minneapolis, USA: University of Minnesota Press; 1952; pp 559-615.

15. Iwata S. Experimental and morphological studies of Manson's tapeworm, Diphyllobothrium erinacei, Rudolphi. Special reference with its scientific name and relationship with Sparganum proliferum, Ijima. Progr Med Parasitol Jpn 1972; 4: 536-590.

16. Lee SH, Chai JY, Seo BS, Cho SY. Two cases of human infection by adult of Spirometra erinacei. Korean J Parasitol 1984; 22: 7177.

17. Jeon HK, Park HS, Lee DM, Choe SJ, Kim KH, Huh S, Sohn WM, Chai JY, Eom KS. Human infections with Spirometra decipiens plerocercoids identified by morphologic and genetic analyses in Korea. Korean J Parasitol 2015; 53: 299-305.

18. Jeon HK, Park H, Lee D, Choe S, Kim KH, Sohn WM, Eom KS. Genetic Identification of Spirometra decipiens Plerocercoids in Terrestrial Snakes from Korea and China. Korean J Parasitol 2016; 54: 181-185.

19. Feng C, Jie W, Yuqin Z. Clinical and radiological analyses of 27 cases of brain parasitic diseases. China Modern Doctor. 2014; 52: 48-50.

20. Moon WK, Chang KH, Cho SY, Han MH, Cha SH, Chi JG, Han MC. Cerebral sparganosis: MR imaging versus CT features. Radiology 1993; 188: 751-757.

21. Wong CW, Ho YS. Intraventricular haemorrhage and hydrocephalus caused by intraventricular parasitic granuloma suggesting cerebral sparganosis. Acta Neurochir 1994; 129: 205-208.

22. Kuroiwa T. Plerocercoid in human brain. Fukuoka Acta Med 1951; 43: 275-278.

23. Han SR, Park JK, Kim YI, Son BC. Posterior cerebral artery infarc- 
tion caused by cerebral sparganosis-induced vasculitis. Eur Neurol 2001; 46: 105-107.

24. Deng L, Xiong P, Qian S. Diagnosis and stereotactic aspiration treatment of cerebral sparganosis: summary of 11 cases. J Neurosurg 2011; 114: 1421-1425.

25. Gong C, Liao W, Chineah A, Wang X, Hou BL. Cerebral sparganosis in children: epidemiological, clinical and MR imaging characteristics. BMC Pediatr 2012; 12: 155.

26. Song T, Wang WS, Zhou BR, Mai WW, Li ZZ, Guo HC, Zhou F. $\mathrm{CT}$ and MR characteristics of cerebral sparganosis. AJNR Am J Neuroradiol 2007; 28: 1700-1705.

27. Chang KH, Chi JG, Cho SY, Han MH, Han DH, Han MC. Cerebral sparganosis: analysis of 34 cases with emphasis on CT features. Neuroradiology 1992; 34: 1-8. 
